\title{
Standart olmayan sonlu fark metodu ile dağılımlı mertebeden SVIR modelinin nümerik analizi
}

\author{
Mehmet KOCABIYIK ${ }^{1,2, *}$, Mevlüde YAKIT ONGUN ${ }^{3}$, \\ İlkem TURHAN ÇETINKAYA ${ }^{4}$ \\ ${ }^{1}$ Burdur Mehmet Akif Ersoy Üniversitesi, Fen Edebiyat Fakültesi, Matematik Bölümü, Burdur, Türkiye \\ ${ }^{2}$ Süleyman Demirel Üniversitesi, Fen Bilimleri Enstitüsü, Isparta, Türkiye \\ ${ }^{3}$ Süleyman Demirel Üniversitesi, Fen Edebiyat Fakültesi, Matematik Bölümü, Isparta, Türkiye \\ ${ }^{4}$ Kütahya Dumlupınar Üniversitesi, Fen Edebiyat Fakültesi, Matematik Bölümü, Kütahya, Türkiye
}

Geliș Tarihi (Received Date): 02.11.2020

Kabul Tarihi (Accepted Date): 11.12.2020

$\ddot{\mathbf{O z}}$

Çoğu bilim dalındaki matematiksel modellemelerde diferansiyel denklemler kullanılmaktadır. Ancak genelde kullanılan adi, kısmi ve kesirli mertebeden diferansiyel denklemlerin kullanımı yerine bu çalışmada daha kapsamlı bir diferansiyel denklem olan dă̆llımlı (distributed) mertebeden diferansiyel denklem ele alınmıştır. Bu çalışmada dağllıml mertebeden diferansiyel denklem yardımı ile epidemik model olan SVIR (Susceptible, Vaccinated, Infectious, Recovered) modeli tanımlanmış ve nümerik çözümü standart olmayan sonlu fark metodu (NSFD) ile araştırllmıştır. Bulaşıcı hastalıkların incelenmesinde kullanılan bu model aynı zamanda içinde barındırdı $\breve{g} l V$ terimi ile hastalık evresinde aşılamanın etkisini ve gelişimini ortaya koymaktadır. Dağılımlı mertebeden diferansiyel denklemlerin kullanılmasında ki temel düşünce hem bu tip denklemlerin bir nevi adi ve kesirli diferansiyel denklemlerin genel hali olmasi hem de içinde tanımlanan yoğunluk fonksiyonu ile farkl durumlar hakkında tek bir denklem ile yorum yaplabilmesindendir. SVIR modelinin nümerik çözümü ve analizi çalışma içerisinde yapılmış ve sonrasında ayrılaştırılmış sisteme ait kararlılık analizi ifade edilmiştir. Bu çalışmalar neticesinde dağllımlı mertebeden modellemenin bu tip epidemik modellemelerde kullanımının mümkün olduğu görülmüş̧ür.

Anahtar kelimeler: Dağılımlı mertebeden diferansiyel denklemler, SVIR epidemik model, aşılama.

\footnotetext{
* Mehmet KOCABIYIK, mkocabiyik@ mehmetakif.edu.tr, http://orcid.org/0000-0002-7701-6946 Mevlüde YAKIT ONGUN, mevludeyakit@sdu.edu.tr, http://orcid.org/0000-0003-2363-9395 İlkem TURHAN ÇETINKAYA, ilkem.turhan@dpu.edu.tr, http://orcid.org/0000-0002-5520-310X
} 


\title{
Numerical analysis of distributed order SVIR model by nonstandard finite difference method
}

\begin{abstract}
Differential equations are used in mathematical modelling in many disciplines of science. In this study, distributed order differential equations which are more comprehensive are considered instead of the ordinary, partial and fractional order differential equations. SVIR (Susceptible, Vaccinated, Infectious, Recovered) model which is an epidemic model is defined with the help of distributed order differential equation and numerical solution is investigated by (NSFD). The model used in examination of contagious disease exhibits the effect and improvement of vaccination with the term $V$. The basic idea of the usage of distributed order differential equations is that these kinds of differential equations are the general case of ordinary and partial differential equations in some way and these equations leads to make interpretation with an only equation about different cases with the help of the intensity function included. Numerical solution and analysis of SVIR model are done in the study and later on, stability analysis of the discrete system is presented. In consequence of these studies, it is seen that the usage of the distributed order modelling is possible for this kind of epidemic modelling.
\end{abstract}

Keywords: Distributed order differential equations, SVIR epidemic model, vaccination.

\section{Giriş}

Aşılama bilindiği üzere kızamık, tetanos, tüberküloz gibi birçok hastalığı kontrol altına almak için kullanılan bir yöntemdir. Bu nedenden dolayı da çözümün farklı evrelerde ve durumlarda etkisini bilmek oldukça önemlidir.

Bulaşıcı hastalıklara ait modelleme ilk olarak Kermack ve Mckendrick tarafindan ortaya konmuştur. Bu çalışmada Kermack ve Mckendrick total popülasyonu SIR (duyarlı, enfekte, iyileşmiş) şeklinde ele almıştır [1]. Sonrasında farklı epidemik modeller için farklı modellemeler kullanılmıştır. Liu vd. ise çalışmasında epidemik modellere aşı stratejilerini de ekleyerek SVIR modelinin oluşturmuştur [2]. Daha sonra bu tip aşılama modelinin çözümü ve analizi hakkında çeşitli çalışmalar yapılmıştır. Örneğin, Kribs ve Velasco çalışmalarında çoklu epidemik modellerde basit aşı modelini ele alıp çözümünü araştırmışlardır [3]. Alexander vd. ise gribin bulaşma anında oluşan dinamikleri için bir aşılama modeli üzerinde çalışmışlardır [4]. Bir diğer çalışmada ise Li vd. aşılama ile epidemik modellerde kararlılık analizi konusunu ele almışlardır [5].

Dağılımlı mertebeden diferansiyel denklemler son senelerde oldukça önem kazanan bir alan olmuştur. Çünkü dağılımlı mertebeden diferansiyel denklemler içinde barındırdığı yoğunluk fonksiyonu ile adi ve kesirli mertebeden diferansiyel denklemler için de bir nevi çözüm oluşturmaktadır. Sadece bu tip denklemlerin genel hali olması nedeniyle değil, yoğunluk fonksiyonunun farklı seçimi ile rahatlıkla farklı durumlarda çözümün nasıl değiştiği görülebilmektedir.

Endemik modellerin farklı evrelerinde duyarlı, iyileşmiş veya enfekte bireyler üzerinde hastalığın gelişim durumunun önceden bilinmesi bireylerin hastalığa karşı vereceği 
tepkiyi ölçmede oldukça önemlidir. Dağılımlı mertebeden diferansiyel denklemlerde tam olarak tek bir denklem ile farklı evreler hakkında bilgi vereceğinden modelin bu tip diferansiyel denklemlerle ele alınması istenen yorum kabiliyetini sağlamaktadır. Bu yorum kabiliyeti ihtiyacından dolayı aşı modeli bu tip diferansiyel denklemler ile ele alınmıştır.

Bu çalışma için, aşı stratejilerine ait Liu vd. [2] tarafindan oluşturulmuş modelin dağılımlı mertebeden hali aşağıdaki şekilde tanımlanmıştır.

$D_{t}^{v(\alpha)} S(t)=a-a S(t)-b S(t) I(t)-c S(t)$

$D_{t}^{v(\alpha)} V(t)=c S(t)-d V(t) I(t)-e V(t)-a V(t)$

$D_{t}^{v(\alpha)} I(t)=b S(t) I(t)+d V(t) I(t)-f I(t)-a I(t)$

$D_{t}^{v(\alpha)} R(t)=e V(t)+f I(t)-a R(t)$

Burada

$S(t)$ : Duyarlı bireylerin zamana bağlı yoğunluk fonksiyonu,

$V(t)$ : Aşılama periyodunda başlayan aşıların zamana bağlı yoğunluk fonksiyonu,

$I(t)$ : Enfekte olmuş bireylerin zamana bağli yoğunluk fonksiyonu,

$R(t)$ : İyileşmiş bireylerin yoğunluk fonksiyonu

olarak sembolize edilmiştir.

Burada, $a$ popülasyonun iyileşme ve doğal ölüm oranı iken $b$ ise duyarlı ve enfekte bireyler arasındaki geçiş katsayısıdır. $c$ parametresi ise duyarlı bireylerin aşılama sürecine geçme oranı olarak tanımlanmıştır. Aşılananların bağışıklık kazanmadan önce enfekte kișilerle temas ettiğinde hastalığın bulașma oranı $d$ ifadesi ile tanımlanmıștır. Aşılananların iyileşmiş popülasyona geçme oranı $e$ iken, $f$ ise enfekte bireylerin iyileşme oranidir.

Epidemik bir model olan SVIR modelinde de bu tip dağılımlı mertebeden diferansiyel denklemlerin kullanımı çözümün farklı aşamalarında yorum kısmında oldukça kolaylık sağlamaktadır. SVIR modelinin çözümü ise içerisinde bulundurduğu V yoğunluk fonksiyonu nedeniyle dikkat çekicidir. Çünkü bu yoğunluk fonksiyonu aşılama evresinde ki takip için kullanılmaktadır.

Yeni tanımlanan ve lineer olmayan (1) sistemi içerisinde bulunan dağılımlı mertebeden türev kavramı ise ilk defa Caputo tarafından tanımlanmış ve sonrasında da yine Caputo bu konu hakkında farklı çalışmalar ile kullanım alanını genişletmiştir [6-9]. Hartley ve Lorenzo ise sürekli dağılımlı mertebeden diferansiyel denklemleri kesirli sistemlerin çözümünde kullanmıştır [10]. Bagley ve Torvik çalışmalarında bu tip denklemlerin çözümünü ve varlığını araştırmıştır [11,12].

Farklı alanlarda kullanımı ile oldukça yaygın hale gelen dağılımlı mertebeden diferansiyel denklemlerin varlık ve tekliğine ait çalışma Ford ve Morgado tarafindan ortaya konmuştur [13]. Nümerik çözümler ile ilgili ise Diethelm ve Ford, Katsikadelis, Li ve Wu gibi yazarların çalışmaları olmuştur [14-16]. Son olarak bu tip denklemlerin kararlılık analizi ile ilgili Aminiknah vd., Najafi vd. ve Refahi vd.'nin çalışmaları bulunmaktadir [17-19]. 
Bu makalede öncelikle 2. Bölümde dağılımlı mertebeden diferansiyel denklemler için temel tanım ve teoremlere yer verilmiştir. Ek olarak yine bu bölümde modelin nümerik çözümü araştırılırken ayrıklaştırma için kullanılacak olan NSFD metoduna ait bazı özet bilgiler bulunmaktadır. 3. Bölümde ise, tanımlanan bu yeni tip dağılımlı mertebeden SVIR modeline ait ayrıklaştırma NSFD metodu yardımı ile yapılmıştır. Kararlılık analizi ile gerekli bilgiler yine bu bölümde verilip sonrasında ayrıklaştırılmış sistemin kararlılık analizine yapılmıştır. Ayrıklaştırılması yapılan sisteme ait nümerik simülasyonlar ve parametre değerleri ile birlikte kararlılık analizi 4. Bölümde yapılmıştır. 5. ve son bölümde ise elde edilen bilgiler ışığında sonuçların değerlendirmesi yer almaktadır.

\section{Bazı temel tanımlar}

Dağılımlı mertebeden diferansiyel denklemler için en önemli ifadelerden biri de kesinlikle kesirli mertebeden türevlerdir. Bu nedenle ilk olarak bazı kesirli türev tanımları, sonrasında ise dağılımlı mertebeden türev ve ayrıklaştırma için kullanılacak NSFD yöntemi hakkında temel bazı tanımlar yer alacaktır. Bölümde değinilen konulara ait detaylı bilgiler için [6,9,20-27] kaynaklarının incelenmesi faydalı olacaktır.

Tanım 2.1: $\alpha$ mertebeden kesirli Caputo türevi,

$D_{C}^{\alpha} f(t)=\frac{1}{\Gamma(n-\alpha)} \int_{\alpha}^{t} \frac{f^{(n)}(z)}{(t-z)^{\alpha-n+1}} d z$

şeklinde tanımlanır. Burada, $n \in \mathbb{N}^{+}$için $n-1<\alpha \leq n$ olmak üzere $f(t),[a, b]$ aralığında integrallenebilir bir fonksiyon ve $\Gamma($.) ise Gamma fonksiyonudur [27].

Tanım 2.2: $n \in \mathbb{N}^{+}$için $n-1<\alpha \leq n$ olmak üzere $g(t),[a, b]$ aralığında integrallenebilir bir fonksiyon olsun.

$$
D_{R L}^{\alpha} g(t)=\frac{1}{\Gamma(n-\alpha)} \frac{d^{n}}{d t^{n}} \int_{\alpha}^{t} \frac{g^{(n)}(z)}{(t-z)^{\alpha-n+1}} d z
$$

şeklinde $\alpha$ mertebeden kesirli Riemann-Liouville türevi tanımlanır [27].

Tanım 2.3: $\mathrm{g}^{(\mathrm{z})}(\mathrm{t})$ fonksiyonu, kapalı $[a, t]$ aralığında sürekli ve $z=1,2, \ldots, n+1$ türevlerine sahip olsun. Bu halde $n<k<n+1$ için Grünwald-Letnikov kesirli türevi,

$D_{G L}^{\alpha} g(t)=\lim _{h \rightarrow 0} h^{-\alpha} \sum_{i=0}^{n}(-1)^{i}\left(\begin{array}{l}k \\ i\end{array}\right) g(t-i h)$

şeklinde tanımlanır [27].

Tanım 2.4: Dağılımlı mertebeden diferansiyel denklemlerin türev tanımı için öncelikle

$D_{t}^{v(\alpha)} f(t)=\int_{\omega_{1}}^{\omega_{2}} v(\alpha) D_{t}^{\alpha} f(t) d \alpha$ 
şeklinde bir integral operatörü tanımlamak gerekir. Bu tanımda $\alpha \epsilon\left(\omega_{1}, \omega_{2}\right)$ olup $\int_{\omega_{1}}^{\omega_{2}} v(\alpha)=l>0$ olmalıdır. Operatörde tanımlı $D_{t}^{\alpha} f(t)$ kesirli türev operatörü olup yerine Caputo, Riemann-Liouville ya da Grünwald-Letnikov kullanılabilir. Operatördeki diğer önemli bir ifade $v(\alpha)$ ise dağılımlı mertebeden türevin yoğunluk fonksiyonudur. $\mathrm{Bu}$ fonksiyonun seçimi adi, kesirli ya da çok daha farklı durumlar için yorum kabiliyeti sağlamaktadır [6-8].

Tanım 2.5: Tanım (2.4) de ifade edilen integral operatör ile dağılımlı mertebeden türevler,

$D_{t}^{v(\alpha)} f(t)=\sum_{i=1}^{n} \alpha^{i} \int_{\omega_{1}}^{\omega_{2}} v_{i}(\alpha) D_{t}^{i-\alpha} f(t) d \alpha+\sum_{j=0}^{n} b_{j} f^{i}(t)$

şeklinde tanımlanır [6-8].

$\mathrm{Bu}$ tanımlardan sonra dağılımlı mertebeden diferansiyel denklemlerin yaklaşık çözümleri için kullanılacak olan yaklaşık Grünwald-Letnikov türev formülü,

$D_{G L}^{\alpha} f(t)=\lim _{h \rightarrow 0} h^{-\alpha} \sum_{i=0}^{n}(-1)^{i}\left(\begin{array}{l}\alpha \\ i\end{array}\right) f(t-i h)$

biçimindedir [21]. Bu tanımda gerekli ayrıklaştırma ve düzenlemeler yapılırsa,

$D_{t}^{\alpha} f(t)=\sum_{i=0}^{n} p_{i}^{\alpha} f\left(t_{n-r}\right), \quad n=1,2,3, \ldots$

denklemi bulunur ki burada $i=1,2,3, \ldots$ için $p_{i}^{\alpha}=\left(1-\frac{1+\alpha}{i}\right) p_{i-1}^{\alpha}, v=h^{-\alpha}$ şeklindedir ve $h$ oldukça küçük seçilmiştir [20,26].

Ayrıklaştırma için kullanılacak olan standart olmayan sonlu fark şemaları ilk olarak Mickens tarafından tanımlanmıştır [22]. Mickens bu şemalar ile klasik nümerik çözüm yöntemlerindeki kararsızlıkları ortadan kaldırmaya çalışmıştır. Bu metotta uygun payda (denominatör) fonksiyonu seçimi ile çıkabilecek negatif çözüm olasılığı ve kararsızlık problemlerinin etkisiz hale getirilebileceğini göstermiştir. Genel olarak NSFD yöntemi için, $\gamma$ : parametre olmak üzere $\frac{d y}{d t}=K(\gamma, y)$ biçimindeki adi diferansiyel denklemi ele alalım. Bu denklem için NSFD şeması aşağıdaki biçimdedir:

$t \rightarrow t_{n}, K(y) \rightarrow K\left(y_{n}\right), \quad y(t) \rightarrow y\left(t_{n}\right), \quad \frac{d y}{d t} \rightarrow \frac{y_{n+1}-y_{n}}{\varphi}$.

$\mathrm{Bu}$ şemada $\varphi$ payda fonksiyonudur ve $\varphi=\frac{1-e^{-p h}}{p}$ şeklinde seçilebilir. Payda fonksiyonunun seçimi için [28] incelenmelidir. Payda fonksiyonu olan $\varphi, h$ adım aralığına ve denge noktası ile hesaplanacak olan $p$ değişkenine bağlıdır. Bu metot ve Grünwald-Letnikov yaklaşık türev formülü ile kesirli mertebeden türevlere de aynı şema uygulanabilir. Sonlu fark şemaları ve NSFD hakkında daha detaylı bilgi için [21-25,2832] kaynaklarına bakılabilir. 


\section{Ayrıklaştırma ve kararlılık analizi}

Bu bölümde, (1) ile tanımlanmış dağılımlı mertebeden diferansiyel denklem sisteminin yaklaşık çözümlerinin bulunabilmesi amacıyla ikinci bölümde ele alınan ve ayrıklaştırma sürecinde gerekli olan standart olmayan sonlu fark şeması ile dağılımlı mertebeden türevler için yaklaşık Grünwald-Letnikov türev tanımı kullanılmıştır. Ek olarak dağılımlı mertebeden diferansiyel denklemlerde oldukça fazla karşılaşılan quadrature formülü ayrıklaştırma içerisinde yer almıştır. Bu bilgiler 1şı̆̆ında (1) diferansiyel denklem sisteminin son hali

$\sum_{k=1}^{Z} \frac{v\left(\alpha_{k}\right)}{Z} \sum_{i=0}^{n+1} p_{i}^{\alpha_{k}} S_{n+1-i}=a-a S_{n+1}-b S_{n+1} I_{n}-c S_{n+1}$

$\sum_{k=1}^{Z} \frac{v\left(\alpha_{k}\right)}{Z} \sum_{i=0}^{n+1} p_{i}^{\alpha_{k}} V_{n+1-i}=c S_{n}-d V_{n+1} I_{n}-e V_{n+1}-a V_{n+1}$

$\sum_{k=1}^{Z} \frac{v\left(\alpha_{k}\right)}{Z} \sum_{i=0}^{n+1} p_{i}^{\alpha_{k}} I_{n+1-i}=b S_{n} I_{n+1}+d V_{n} I_{n+1}-f I_{n+1}-a I_{n+1}$

$\sum_{k=1}^{Z} \frac{v\left(\alpha_{k}\right)}{Z} \sum_{i=0}^{n+1} p_{i}^{\alpha_{k}} R_{n+1-i}=e V_{n}+f I_{n}-a R_{n+1}$

şeklinde olacaktır. Burada, $j=1,2,3,4$ ve $0<\alpha_{k}<1$ için $p_{0}^{\alpha_{k}}=\left(\varphi_{j}(h)\right)^{-\alpha_{k}}$ dir. Standart olmayan sonlu fark şeması içerisinde tanımlanan denominatör (payda) fonksiyonları, aşağıdaki şekilde seçilmiştir:

Burada

$$
\begin{aligned}
& \varphi_{1}(h)=\frac{e^{(a+c) h}-1}{a+c}, \varphi_{2}(h)=\frac{e^{(a+e) h}-1}{a+e}, \\
& \varphi_{3}(h)=\frac{e^{(a+f) h}-1}{a+f}, \varphi_{4}(h)=\frac{e^{a h}-1}{a} .
\end{aligned}
$$

$$
\begin{aligned}
& \sum_{i=0}^{n+1} p_{i}^{\alpha_{k}} S_{n+1-i}=p_{0}^{\alpha_{k}} S_{n+1}+\sum_{i=1}^{n+1} p_{i}^{\alpha_{k}} S_{n+1-i}=\left(\varphi_{1}(h)\right)^{-\alpha_{k}} S_{n+1}+\sum_{i=1}^{n+1} p_{i}^{\alpha_{k}} S_{n+1-i}, \\
& \sum_{i=0}^{n+1} p_{i}^{\alpha_{k}} V_{n+1-i}=p_{0}^{\alpha_{k}} V_{n+1}+\sum_{i=1}^{n+1} p_{i}^{\alpha_{k}} V_{n+1-i}=\left(\varphi_{2}(h)\right)^{-\alpha_{k}} V_{n+1}+\sum_{i=1}^{n+1} p_{i}^{\alpha_{k}} V_{n+1-i}, \\
& \sum_{i=0}^{n+1} p_{i}^{\alpha_{k}} I_{n+1-i}=p_{0}^{\alpha_{k}} I_{n+1}+\sum_{i=1}^{n+1} p_{i}^{\alpha_{k}} I_{n+1-i}=\left(\varphi_{3}(h)\right)^{-\alpha_{k}} I_{n+1}+\sum_{i=1}^{n+1} p_{i}^{\alpha_{k}} I_{n+1-i}, \\
& \sum_{i=0}^{n+1} p_{i}^{\alpha_{k}} R_{n+1-i}=p_{0}^{\alpha_{k}} R_{n+1}+\sum_{i=1}^{n+1} p_{i}^{\alpha_{k}} R_{n+1-i}=\left(\varphi_{4}(h)\right)^{-\alpha_{k}} R_{n+1}+\sum_{i=1}^{n+1} p_{i}^{\alpha_{k}} R_{n+1-i},
\end{aligned}
$$

düzenlemeleri her bir denklem için yapıldıktan sonra (2) sisteminde bu ifadelerin yerine yazılması ve yalnız bırakma işleminden sonra denklemin ayrıklaştırılmış olarak son hali,

$$
\begin{aligned}
& S_{n+1=}=\frac{a-H\left(p_{1}^{\alpha_{k}} S_{n}+\sum_{i=2}^{n+1} p_{i}^{\alpha_{k}} S_{n+1-i}\right)}{\left(\left(U_{1}\right)^{-\alpha_{k}}+a+c+b I_{n}\right)}, \\
& V_{n+1=}=\frac{c S_{n}-H\left(p_{1}^{\alpha_{k}} V_{n}+\sum_{i=2}^{n+1} p_{i}^{\alpha_{k}} V_{n+1-i}\right)}{\left(\left(U_{2}\right)^{\left.-\alpha_{k}+a+e+d I_{n}\right)}\right.}, \\
& I_{n+1}=\frac{-H\left(p_{1}^{\alpha_{k}} I_{n}+\sum_{i=2}^{n+1} p_{i}^{\alpha_{k}} I_{n+1-i}\right)}{\left(\left(U_{3}\right)^{\left.-\alpha_{k}+a+f-b S_{n}-d V_{n}\right)},\right.} \\
& R_{n+1}=\frac{e V_{n}+f I_{n}-H\left(p_{1}^{\alpha_{k}} R_{n}+\sum_{i=2}^{n+1} p_{i}^{\alpha_{k}} R_{n+1-i}\right)}{\left(\left(U_{4}\right)^{-\alpha_{k}}+a\right)}
\end{aligned}
$$

şeklinde olur. Burada, $j=1,2,3,4$ için $\sum_{k=1}^{Z} \frac{v\left(\alpha_{k}\right)}{Z}=H$ ve $\sum_{k=1}^{Z} \frac{v\left(\alpha_{k}\right)}{Z}\left(\varphi_{j}(h)\right)^{-\alpha_{k}}=U_{j}$ olarak kullanılmıştır. 
Teorem 3.1. Ayrıklaştırılmış (3) sisteminde pozitif başlangıç koşulları ve pozitif parametrelerin seçimi ile aşă̆ıdaki özellikler sağlanırsa elde edilecek sonuçlar pozitif olmaktadır.

$$
\begin{array}{ll}
\text { i) } & a>H\left(p_{1}^{\alpha_{k}} S_{n}+\sum_{i=2}^{n+1} p_{i}^{\alpha_{k}} S_{n+1-i}\right) \\
\text { ii) } & c S_{n}>H\left(p_{1}^{\alpha_{k}} V_{n}+\sum_{i=2}^{n+1} p_{i}^{\alpha_{k}} V_{n+1-i}\right) \\
\text { iii) } & \left(U_{3}\right)^{-\alpha_{k}}+a+f<b S_{n}+d V_{n} \\
\text { iv) } & e V_{n}+f I_{n}>H\left(p_{1}^{\alpha_{k}} R_{n}+\sum_{i=2}^{n+1} p_{i}^{\alpha_{k}} R_{n+1-i}\right)
\end{array}
$$

\section{İspat}

Pozitif başlangıç koşulları ve pozitif parametreler seçimlerine ek olarak $\frac{a-H\left(p_{1}^{\alpha_{k}} S_{n}+\sum_{i=2}^{n+1} p_{i}^{\alpha_{k}} S_{n+1-i}\right)}{\left(\left(U_{1}\right)^{-\alpha} k_{k}+a+c+b I_{n}\right)}>0$ olması için $a>H\left(p_{1}^{\alpha_{k}} S_{n}+\sum_{i=2}^{n+1} p_{i}^{\alpha_{k}} S_{n+1-i}\right)$ koşulu sağlanmalıdır. $V_{n+1}$ teriminin pozitifliği için ayrıklaştırılmış halinde payda kısmının pozitif olduğu görüldüğünden pay kısmının negatifliği için $c S_{n}>H\left(p_{1}^{\alpha_{k}} V_{n}+\right.$ $\left.\sum_{i=2}^{n+1} p_{i}^{\alpha_{k}} V_{n+1-i}\right)$ ifadesi gerçekleşmelidir. Sırasıyla elde edilen $I_{n+1}$ ve $R_{n+1}$ terimlerinin pozitifliği için ise $\left(U_{3}\right)^{-\alpha_{k}}+a+f<b S_{n}+d V_{n}$ ve $e V_{n}+f I_{n}>H\left(p_{1}^{\alpha_{k}} R_{n}+\right.$ $\left.\sum_{i=2}^{n+1} p_{i}^{\alpha_{k}} R_{n+1-i}\right)$ ifadeleri sağlandığında pozitif başlangıç koşulları ve parametreler ile çözümler de pozitif olmaktadır.

Şimdi modelin kararlılık analizi kısmında lazım olacak olan J Jacobian matrisi, (3) ayrıklaştırılmış sistemi için:

$J(S, V, I, R)=\left(\begin{array}{cccc}j_{11} & 0 & j_{13} & 0 \\ j_{21} & j_{22} & j_{23} & 0 \\ j_{31} & j_{32} & j_{33} & 0 \\ 0 & j_{42} & j_{43} & j_{44}\end{array}\right)$

biçimindedir ki burada

$$
\begin{aligned}
& j_{11}=\frac{-H p_{1}^{\alpha_{k}}}{\left(\left(U_{1}\right)^{-\alpha_{k}}+a+c+b I_{n}\right)^{\prime}} \\
& j_{13}=-b\left(\frac{a-H\left(p_{1}^{\alpha_{k}} S_{n}+\sum_{i=2}^{n+1} p_{i}^{\alpha_{k}} S_{n+1-i}\right)}{\left(\left(U_{1}\right)^{-\alpha_{k}}+a+c+b I_{n}\right)^{2}}\right) \text {, } \\
& j_{21}=\frac{c}{\left(\left(U_{2}\right)^{-\alpha_{k}}+a+e+d I_{n}\right)}, \quad j_{22}=\frac{-H p_{1}^{\alpha_{k}}}{\left(\left(U_{2}\right)^{-\alpha_{k}}+a+e+d I_{n}\right)^{\prime}}, \\
& j_{23}=-d\left(\frac{c S_{n}-H\left(p_{1}^{\alpha} V_{n}+\sum_{i=2}^{n+1} p_{i}^{\alpha} k_{n+1-i}\right)}{\left(\left(U_{2}\right)^{-\alpha} k+a+e+d I_{n}\right)^{2}}\right) \text {, } \\
& j_{31}=b\left(\frac{-H\left(p_{1}^{\alpha_{k}} I_{n}+\sum_{i=2}^{n+1} p_{i}^{\alpha_{k}} I_{n+1-i}\right)}{\left(\left(U_{3}\right)^{-\alpha} k+a+f-b S_{n}-d V_{n}\right)^{2}}\right), j_{32}=d\left(\frac{-H\left(p_{1}^{\alpha_{k}} I_{n}+\sum_{i=2}^{n+1} p_{i}^{\alpha_{k}} I_{n+1-i}\right)}{\left(\left(U_{3}\right)^{-\alpha} k+a+f-b S_{n}-d V_{n}\right)^{2}}\right) \text {, } \\
& j_{33}=\frac{-H p_{1}^{\alpha_{k}}}{\left(\left(U_{3}\right)^{-\alpha} k+a+f-b S_{n}-d V_{n}\right)}, \quad j_{42}=\frac{e}{\left(\left(U_{4}\right)^{-\alpha}+a\right)}, j_{43}=\frac{f}{\left(\left(U_{4}\right)^{\left.-\alpha_{k}+a\right)}\right.}, \quad j_{44}=\frac{-H p_{1}^{\alpha_{k}}}{\left(\left(U_{4}\right)^{\left.-\alpha_{k}+a\right)}\right.} \text {. }
\end{aligned}
$$

Kararlılık analizini araştıracağımız denge noktasını bulmak için,

$$
\frac{a-H\left(p_{1}^{\alpha_{k}} S_{n}+\sum_{i=2}^{n+1} p_{i}^{\alpha_{k}} S_{n+1-i}\right)}{\left(\left(U_{1}\right)^{\left.-\alpha_{k}+a+c+b I_{n}\right)}\right.}=S_{n}
$$




$$
\begin{aligned}
& \frac{c S_{n}-H\left(p_{1}^{\alpha_{k}} V_{n}+\sum_{i=2}^{n+1} p_{i}^{\alpha_{k}} V_{n+1-i}\right)}{\left(\left(U_{2}\right)^{-\alpha} k+a+e+d I_{n}\right)}=V_{n} \\
& \frac{-H\left(p_{1}^{\alpha_{k}} I_{n}+\sum_{i=2}^{n+1} p_{i}^{\alpha_{k}} I_{n+1-i}\right)}{\left(\left(U_{3}\right)^{-\alpha} k+a+f-b S_{n}-d V_{n}\right)}=I_{n} \\
& \frac{e V_{n}+f I_{n}-H\left(p_{1}^{\alpha_{k}} R_{n}+\sum_{i=2}^{n+1} p_{i}^{\alpha_{k}} R_{n+1-i}\right)}{\left(\left(U_{4}\right)^{-\alpha} k+a\right)}=R_{n}
\end{aligned}
$$

sisteminin çözülmesi gereklidir. İlk olarak (7) denkleminin çözümü araştırıldığında pozitif parametreler nedeniyle çözümün ancak $I_{n}=0$ durumunda sağlanacağ 1 görülmektedir. Elde edilen bu bilgi ile (5) denkleminde gerekli düzenlemeler ile $S_{n}=$

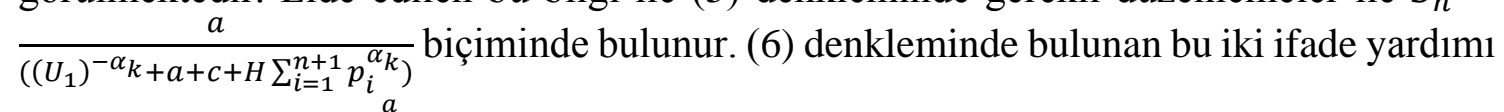
ile $V_{n}=\frac{c \frac{a}{\left(\left(U_{1}\right)^{-\alpha} k+a+c+H \sum_{i=1}^{n+1} p_{i}^{\alpha_{k}}\right)}}{\left(\left(U_{2}\right)^{-\alpha} k+a+e+H \sum_{i=1}^{n+1} p_{i}^{\alpha_{k}}\right)}$ şeklinde bulunur. Son olarak (8) denkleminde ki

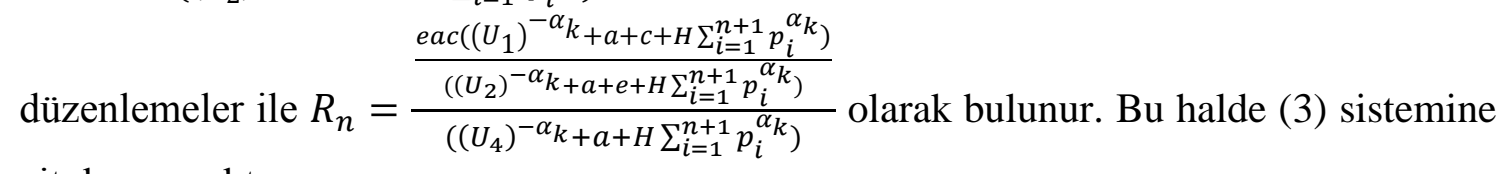
ait denge noktasi:

$$
=\left(\frac{D=(S, V, I, R)}{\left(\left(U_{1}\right)^{-\alpha_{k}}+a+c+H \sum_{i=1}^{n+1} p_{i}^{\alpha_{k}}\right)}, \frac{c \frac{a}{\left(\left(U_{1}\right)^{-\alpha_{k}}+a+c+H \sum_{i=1}^{n+1} p_{i}^{\alpha_{k}}\right)}}{\left(\left(U_{2}\right)^{-\alpha_{k}}+a+e+H \sum_{i=1}^{n+1} p_{i}^{\alpha_{k}}\right)}, 0, \frac{\frac{e a c\left(\left(U_{1}\right)^{-\alpha_{k}}+a+c+H \sum_{i=1}^{n+1} p_{i}^{\alpha_{k}}\right)}{\left(\left(U_{2}\right)^{-\alpha_{k}}+a+e+H \sum_{i=1}^{n+1} p_{i}^{\alpha_{k}}\right)}}{\left(\left(U_{4}\right)^{-\alpha_{k}}+a+H \sum_{i=1}^{n+1} p_{i}^{\alpha_{k}}\right)}\right)
$$

olarak hesaplanır.

Tanım 3.1: $\frac{d v}{d t}=F(v)$ denkleminin yaklaşık çözümü $v\left(t_{k}\right)$ olsun. Bu çözüme ait $h$ adım aralığına göre genel nümerik şeması:

$G_{h}\left(v_{k}\right)=Q_{h}\left(F ; v_{k}\right), v_{k} \approx v\left(t_{k}\right), \quad t_{k}=t_{0}+k h$

biçimindedir. Burada $G_{h}\left(v_{k}\right) \approx\left(\frac{d v_{1}}{d t}, \frac{d v_{2}}{d t}, \ldots, \frac{d v_{n}}{d t}\right)^{T}$ ve $Q_{h}\left(F ; v_{k}\right)$ ise yaklaşık çözümdür $[33,34]$.

Lemma 3.1. D noktasl (9) sistemine ait denge noktasl olsun. Bu halde denge noktasinın kararlı olması için Jacobian matrisinde yerine koyulduğunda elde edilen tüm öz değerlerin mutlak değerleri 1'den küçük olmalıdır. Aksi halde en az bir öz değerin mutlak değeri l'den küçük değil ise denge noktası kararlı değildir. Ek olarak denge noktası kararlı iken eğer tüm öz değerler sıfirdan küçük ise denge noktası lokal asimptotik kararlıdır [33,35].

Bazı durumlarda karalılık analizinde öz değerleri bulmak kolay olmamaktadır. Bu nedenle öz değerler için bulunacak olan karakteristik denklemin katsayıları ile tüm $\lambda$ değerlerinin mutlak değerlerinin 1'den küçük olup-olmayacağı belirlenebilir. Bunun için Schur-Cohn testi karakteristik denklemin katsayıları yardımı ile uygulanır. Bu test için

$$
P(\lambda)=t_{n} \lambda^{n}+t_{n-1} \lambda^{n-1}+\cdots+t_{2} \lambda^{2}+t_{1} \lambda+t_{0}
$$


karakteristik polinomunu ele alalım. Bu katsayılar ile $t_{n}>0$ olmak üzere,

$$
\begin{aligned}
& s_{2, i}=\operatorname{det}\left(\begin{array}{cc}
t_{0} & t_{n-i} \\
t_{n} & t_{i}
\end{array}\right), \quad i=0,1, \ldots, n-1 . \\
& s_{3, i}=\operatorname{det}\left(\begin{array}{cc}
s_{2,0} & s_{2, n-1-i} \\
s_{2, n-1} & s_{2, i}
\end{array}\right), \quad i=0,1, \ldots, n-2 . \\
& \ldots \\
& s_{n, i}=\operatorname{det}\left(\begin{array}{cc}
s_{n-1,0} & s_{n-1,3-i} \\
s_{n-1,3} & s_{n-1, i}
\end{array}\right), \quad i=0,1,2 .
\end{aligned}
$$

ifadeleri tanımlansın.

Schur-Cohn testine göre tüm öz değerlerin mutlak değerlerinin 1'den küçük olması için aşağıdaki koşullar sağlanmalıdır;

$$
\begin{array}{ll}
\text { i) } & P(1)>0, \\
\text { ii) } & (-1)^{n} P(-1)>0, \\
\text { iii) } & \left|t_{0}\right|<t_{n}, \\
\text { iv) } & \left|s_{i, 0}\right|>\left|s_{i, n+1-i}\right|, i=2,3, \ldots, n-1,[20,36] .
\end{array}
$$

Lemma 3.2. $P(\lambda)=\lambda^{4}+t_{3} \lambda^{3}+t_{2} \lambda^{2}+t_{1} \lambda+t_{0},\left(t_{4}=1\right)$ şeklindeki karakteristik denklem için tüm öz değerlerin mutlak değerlerinin 1'den küçük olmasl:

$$
\begin{array}{ll}
\text { i) } & 1+t_{3}+t_{2}+t_{1}+t_{0}>0, \\
\text { ii) } & 1+t_{3}+t_{2}+t_{1}+t_{0}>0, \\
\text { iii) } & \left|t_{0}\right|<1 \\
\text { iv) } & \left|s_{2,0}\right|>\left|s_{2,3}\right|, \\
\text { v) } & \left|s_{3,0}\right|>\left|s_{3,2}\right|,
\end{array}
$$

ifadelerinin sağlanması ile mümkündür [20,36].

Ek olarak bu tür epidemik sistemlerde nüfusun durumunu kontrol etmek amaciyla sınır koşulları oldukça önemlidir. Bu sebeple temel üreme sayısı olarak da adlandırılan $R_{0}$ sayısını bulmak kararlılık açısından gereklidir. $R_{0}$ sayısını bulmak için öncelikle elde edilen Jacobian matrisi $J=P-S$ formatında iki matrisin farkı olarak ifade edilir. Daha sonra elde edilen bu matrisler ile $P S^{-1}$ matrisi yazılır ve determinantı elde edilirse (1) sistemine ait temel üreme sayısı;

$$
R_{0}=\frac{a c d(a+e)}{c(a+f)+a(-b+f+a)}
$$

şeklinde bulunur [37].

Teorem 3.2. $R_{0}>1$ için aşă̆ıldaki koşulların sağlanması durumunda, (3) sisteminin $D$ denge noktası lokal asimptotik kararlıdır, aksi halde kararsızdır.

$$
\begin{aligned}
& \text { i) } \quad\left|-H p_{1}^{\alpha_{k}}\right|<\left|\left(\left(U_{1}\right)^{-\alpha_{k}}+a+c\right)\right| \\
& \text { ii) } \quad\left|-H p_{1}^{\alpha_{k}}\right|<\left|\left(\left(U_{2}\right)^{-\alpha_{k}}+a+e\right)\right|
\end{aligned}
$$

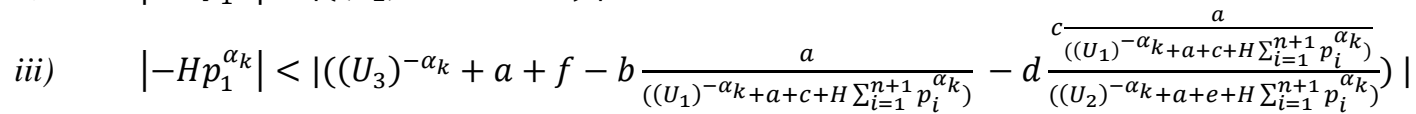

$$
\begin{aligned}
& \text { iv) } \quad\left|-H p_{1}^{\alpha_{k}}\right|<\left|\left(\left(U_{4}\right)^{-\alpha_{k}}+a\right)\right|
\end{aligned}
$$




\section{İspat}

$D$ olarak ifade edilmiş denge noktası (4) ile ifade edilen Jacobian matrisinde yerine yazılırsa;

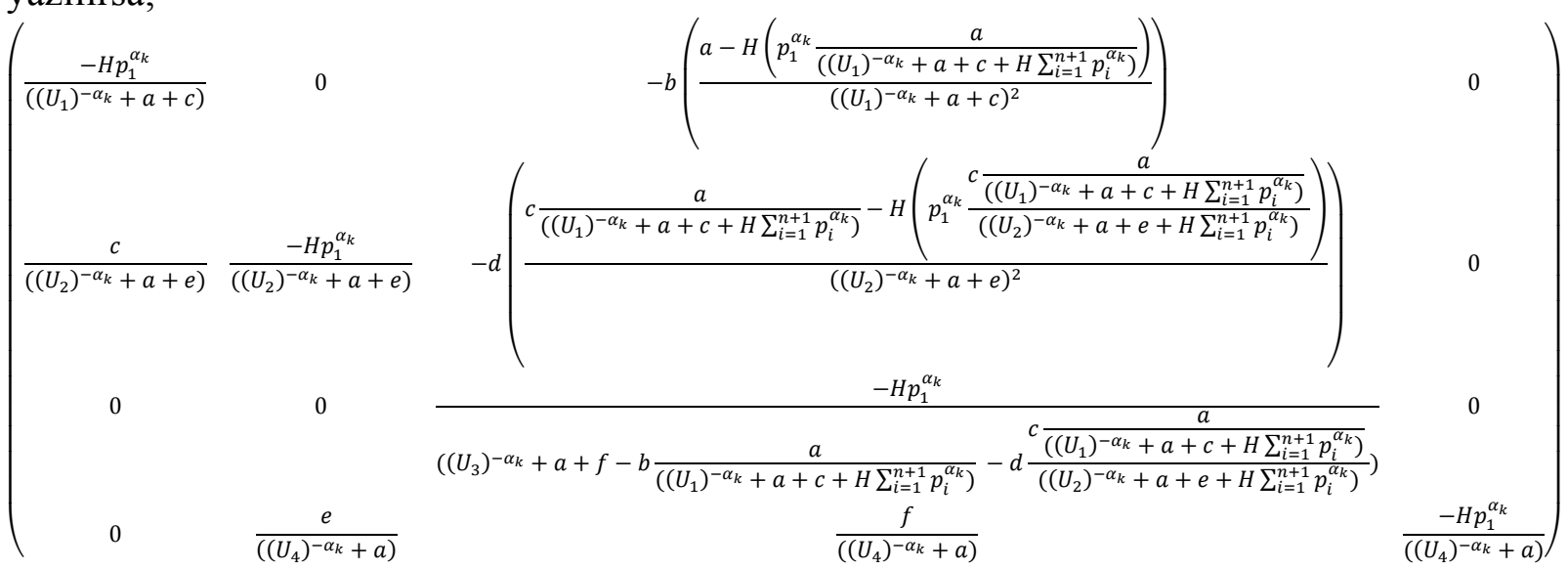

elde edilir. Elde edilen bu matrisin $|J-\lambda I|=0$ determinantı kullanılarak öz değerleri

$$
\begin{aligned}
& \lambda_{\mathbf{1}}=\frac{-H p_{1}^{\alpha_{k}}}{\left(\left(U_{1}\right)^{-\alpha_{k}}+a+c\right)}, \quad \lambda_{\mathbf{2}}=\frac{-H p_{1}^{\alpha_{k}}}{\left(\left(U_{2}\right)^{-\alpha_{k}}+a+e\right)}, \quad \lambda_{\mathbf{3}}=\frac{-H p_{1}^{\alpha_{k}}}{\left(\left(U_{4}\right)^{-\alpha_{k}}+a\right)^{\prime}} \\
& \lambda_{4}=\frac{-H p_{1}^{\alpha_{k}}}{\left(\left(U_{3}\right)^{-\alpha_{k}}+a+f-b \frac{a}{\left(\left(U_{1}\right)^{-\alpha_{k}}+a+c+H \sum_{i=1}^{n+1} p_{i}^{\alpha_{k}}\right)}-d \frac{\left.a \frac{\left.a+c+H \sum_{i=1}^{n+1} p_{i}^{\alpha_{k}}\right)}{\left(\left(U_{1}\right)^{-\alpha_{k}}+a+c \sum_{i=1}^{\alpha_{k}}\right)}\right)}{\left(\left(U_{2}\right)^{-\alpha_{k}}+a+e+H \sum_{i}^{n+1} p^{\alpha_{k}}\right)} .\right.}
\end{aligned}
$$

olarak bulunur.

Burada fark edildiği üzere $\lambda_{4}$ harici diğer tüm öz değerlerin pozitif parametreler kullanıldığında negatif olduğu aşikardır. $\lambda_{4}$ için ise $R_{0}$ ifadesi 1 'den büyük kabul edilirse yani $a c d(a+e)>c(a+f)+a(-b+f+a)$ olursa, bu halde $\lambda_{4}$ ifadesinin paydas 1 pozitif olmak durumunda kalır ve üst kısmın negatif olmasından dolayı $\lambda_{4}$ öz değeri negatif olarak bulunur. Tüm $\lambda$ değerlerinin mutlak değerleri payda kısımlarının pay kısımlarından daha büyük olması gerektiğinden dolayı belirtilen (i)-(iv) koşullarının sağlanması durumunda da D denge noktası lokal asimptotik kararlıdır.

\section{Nümerik simülasyon}

Bu bölümde ayrıklaştırılmıș (3) diferansiyel denklem sistemi için nümerik simülasyonlar verilmiștir. Ek olarak D denge noktasındaki kararlılık analizi aşağıda verilen parametre değerleri ile araştırılmıştır. Öncelikle başlangıç değerleri $S(0)=0.4, V(0)=0.4$, $I(0)=0.2$ ve $R(0)=0.1$ olarak alınmış ve parametreler ise $a=1, b=15, c=15, d=$ $10, e=4$ ve $f=2$ şeklinde seçilmiştir [37]. Bu seçimler ile $R_{0}=22,272727>1$ olarak bulunmuştur. Öncelikle (3) ayrıklaştırılmış sisteminde bu verilerin yerleştirilmesi ve $v(\alpha)=1, \alpha=0.4$ ve $h=0.8$ seçilmesiyle sisteme ait çözüm grafikleri Şekil 1'de verilmiştir. Sonrasında farklı $v(\alpha)$ değerleri için tüm sistemdeki fonksiyonların grafikleri ayr1 ayr1 Şekil 2- Şekil 5' de gösterilmiştir. İfade edilen değerler ile denge noktas1 $D(0.062499,0.937428,0,3.749891)$ şeklinde elde edilmiş ve Jacobian matrisinde bu noktanın yerine yazılmasıyla sistemin karakteristik denklemi 
$P(\lambda)=\lambda^{4}+t_{3} \lambda^{3}+t_{2} \lambda^{2}+t_{1} \lambda+t_{0}$

biçiminde olup buradaki katsayılar

$t_{3}=0.012887, t_{2}=0.272094 .10^{-4}, t_{1}=0.114651 .10^{-7}$ ve $t_{0}=0.103291 .10^{-11}$

olarak elde edilir. Schur-Cohn kriterine göre

i) $\quad 1+t_{3}+t_{2}+t_{1}+t_{0}=1.012191>0$,

ii) $\quad 1-t_{3}+t_{2}-t_{1}+t_{0}=0.987139>0$,

iii) $\quad\left|t_{0}\right|=0.103291 .10^{-11}<1$,

iv) $\quad\left|s_{2,0}\right|=1>\left|s_{2,3}\right|=0.114651 \cdot 10^{-7}$,

v) $\quad\left|s_{3,0}\right|=1>\left|s_{3,2}\right|=0.272093 \cdot 10^{-4}$,

ifadeleri sağlanır. Bu durumda tüm $\lambda^{\prime}$ lar için $|\lambda|<1$ sağlandığından D kararlı bir denge noktasıdır. Ek olarak karakteristik denklemin çözümü ile

$\lambda_{1}=-0.00012, \lambda_{2}=-0.010369, \lambda_{3}=-0.001993, \quad \lambda_{4}=-0.000399$.

şeklinde elde edilmiş olup tüm öz değerler sıfırdan küçük olduğundan D denge noktası lokal asimptotik kararlıdır.

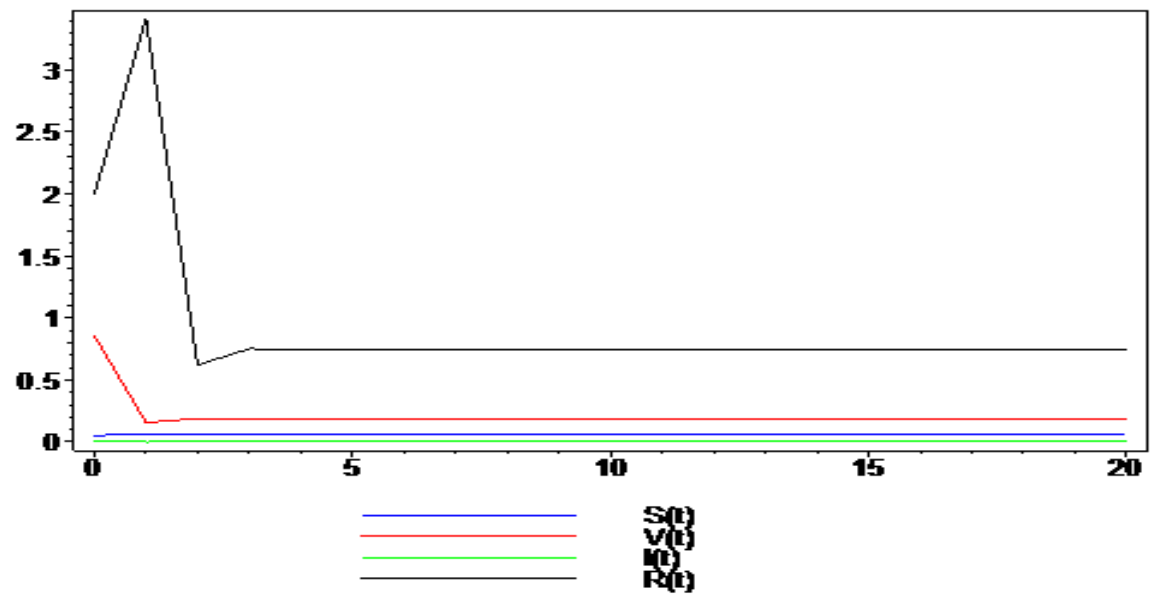

Şekil 1. $v(\alpha)=1$ için SVIR sisteminin çözüm grafikleri.

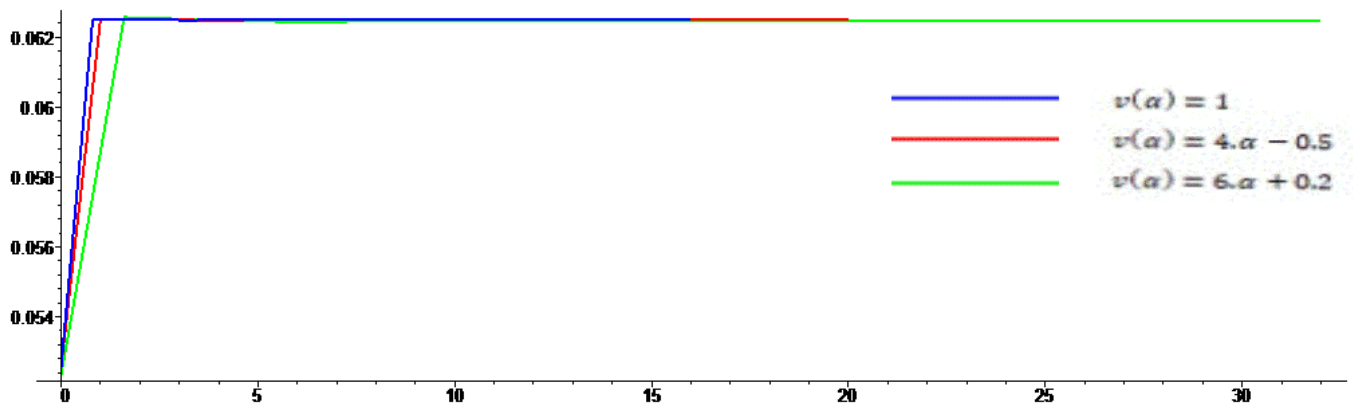

Şekil 2. Farklı $\mathrm{v}(\alpha)$ değerleri için $S(t)$ fonksiyonu grafikleri. 


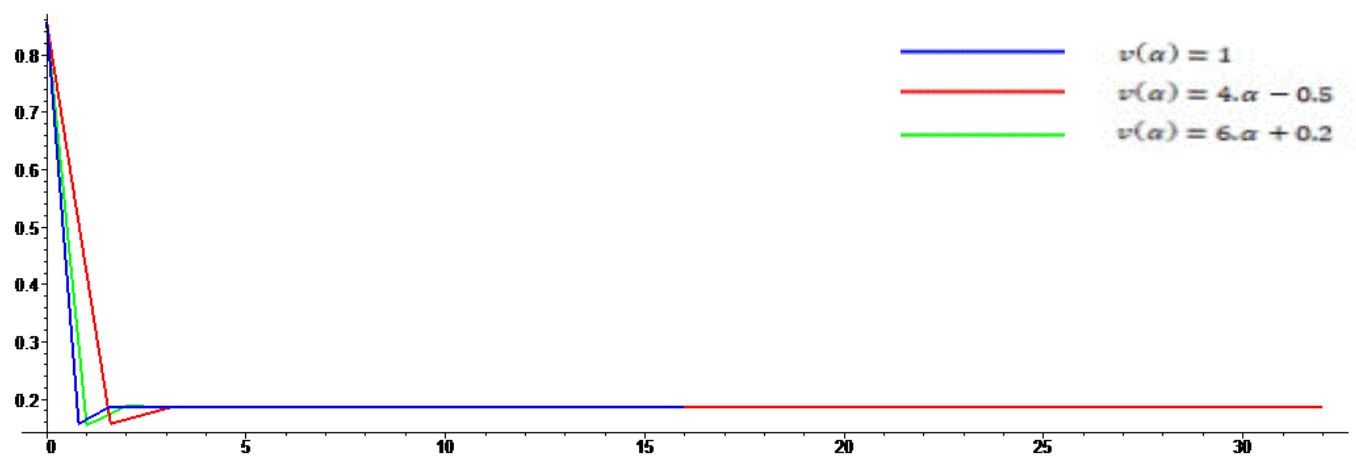

Şekil 3. Farklı $v(\alpha)$ değerleri için $V(t)$ fonksiyonu grafikleri.

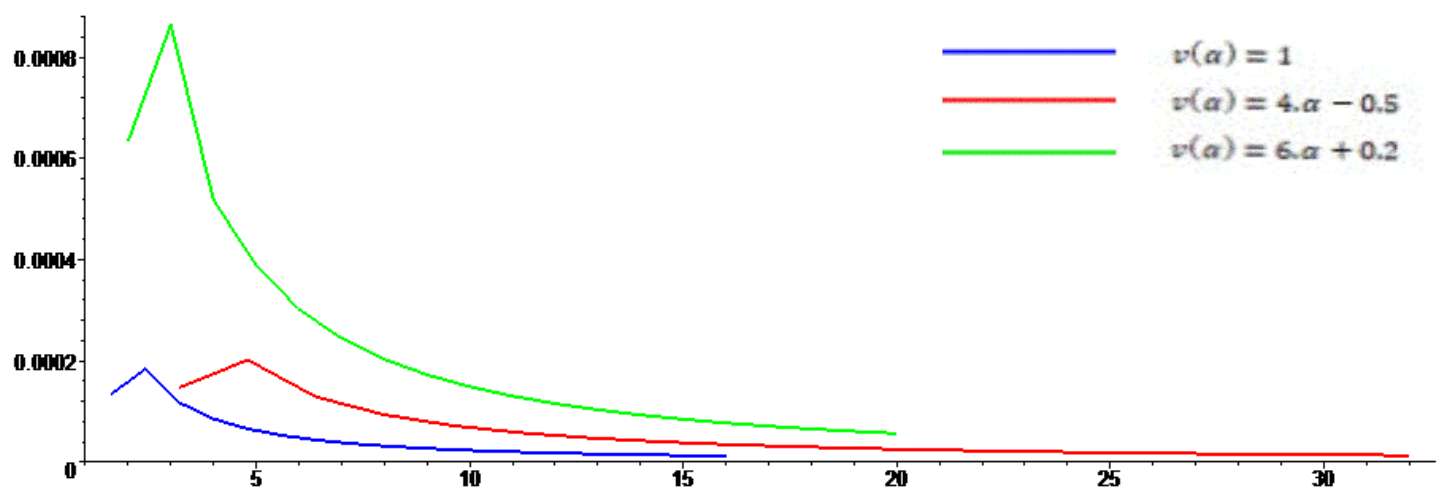

Şekil 4. Farklı v( $\alpha)$ değerleri için $I(t)$ fonksiyonu grafikleri.

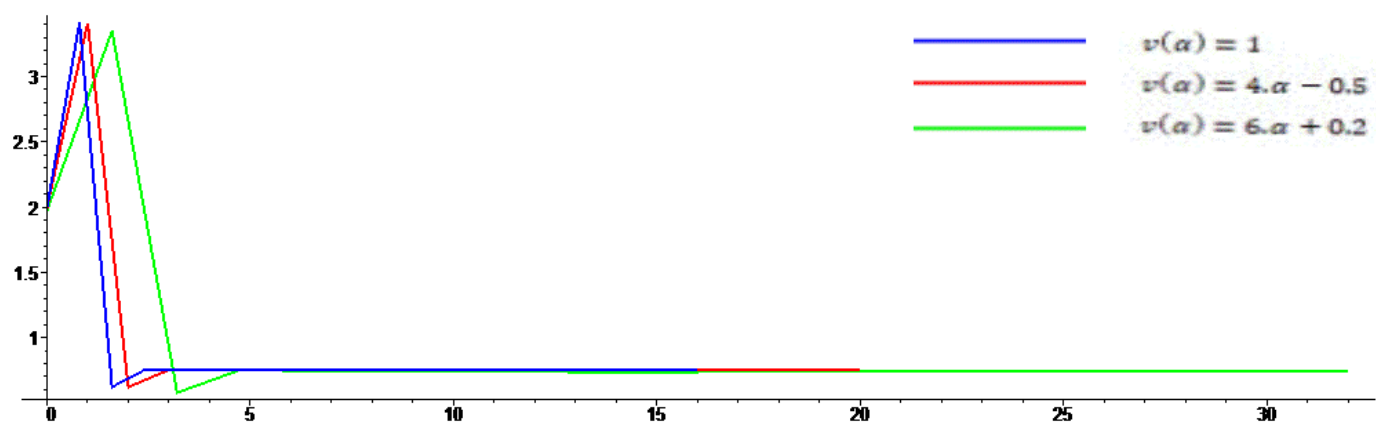

Şekil 5. Farklı $\mathrm{v}(\alpha)$ değerleri için $R(t)$ fonksiyonu grafikleri.

\section{Sonuç}

$\mathrm{Bu}$ çalışmada öncelikler aşı stratejilerini de içeren epidemik bir model olan SVIR modelinin dağılımlı mertebeden hali yeni bir model olarak ifade edilmiştir. Sonrasında yeni tanımlanan bu denklemin ayrıklaştırılması ve kararlılık analizi yapılmıştır. Yapılan bu çalışmalarla ilgili de son olarak 4. Bölümde nümerik simülasyonlar verilmiştir. Elde edilen bulgular ışı̆̆ında dağılımlı mertebeden diferansiyel denklemlerin bu tip epidemik modellerde kullanılmasının oldukça önemli olduğu görülmüştür. Çünkü dağılımlı 
mertebeden denklemlerin içerdiği $\mathrm{v}(\alpha)$ değeri ile yorum ve analiz kısmında tek bir denklemle birden fazla denklem tipine ait yorum kabiliyeti geliştirildiği görülmüştür. Bu çalışma ile dağılımlı mertebeden diferansiyel denklemlerin nümerik çözümleri için NSFD şemaları ile ayrıklaştırma işleminin yapılmasının ve çözümlerin kararlılık analizinin mümkün olduğu görülmüştür.

\section{Teşekkür}

Yazarlardan Mehmet KOCABIYIK, 2211-E Programı ile maddi ve manevi destek veren Türkiye Bilimsel ve Teknolojik Araştırma Kurumuna (TÜBİTAK) teşekkür eder.

\section{Kaynaklar}

[1] Kermark, M. ve Mckendrick, A. Contributions to the mathematical theory of epidemics. Part I. Proceedings of the royal society A, 115(5), 700-721, (1927).

[2] Liu, X., Takeuchi, Y. ve Iwami, S. SVIR epidemic models with vaccination strategies. Journal of Theoretical Biology, 253(1), 1-11, (2008).

[3] Kribs-Zaleta, C. M. ve Velasco-Hernández, J. X. A simple vaccination model with multiple endemic states. Mathematical biosciences, 164(2), 183-201, (2000).

[4] Alexander, M. E., Bowman, C., Moghadas, S. M., Summers, R., Gumel, A. B. ve Sahai, B. M. A vaccination model for transmission dynamics of influenza. SIAM Journal on Applied Dynamical Systems, 3(4), 503-524, (2004).

[5] Li, J., Yang, Y. ve Zhou, Y. Global stability of an epidemic model with latent stage and vaccination. Nonlinear Analysis: Real World Applications, 12(4), 2163-2173, (2011).

[6] Caputo, M. Elasticita e dissipazione. Zanichelli, (1969).

[7] Caputo, M. Mean fractional-order-derivatives differential equations and filters. Annali dell'Universita di Ferrara, 41(1), 73-84, (1995).

[8] Caputo, M. Distributed order differential equations modelling dielectric induction and diffusion. Fractional Calculus and Applied Analysis, 4(4), 421-442, (2001).

[9] Caputo, M. Diffusion with space memory modelled with distributed order space fractional differential equations. Annals of Geophysics, 223-234, (2003).

[10] Hartley, T. T. ve Lorenzo, C. F. Fractional-order system identification based on continuous order distributions. Signal processing, 83(11), 2287-2300, (2003).

[11] Bagley, R. L. ve Torvik, P. J. On the existence of the order domain and the solution of distributed order equations-Part I. International Journal of Applied Mathematics, 2(7), 865-882, (2000).

[12] Bagley, R. L. ve Torvik, P. J. On the existence of the order domain and the solution of distributed order equations-Part II. International Journal of Applied Mathematics, 2(8), 965-988, (2000).

[13] Ford, N. J. ve Morgado, M. L. Distributed order equations as boundary value problems. Computers and Mathematics with Applications, 64(10), 2973-2981, (2012).

[14] Diethelm, K. ve Ford, N. J. Numerical analysis for distributed order differential equations. Journal of Computational and Applied Mathematics, 225(1), 96104, (2009). 
[15] Katsikadelis, J. T. Numerical solution of distributed order fractional differential equations. Journal of Computational Physics, 259, 11-22, (2014).

[16] Li, X. Y., ve Wu, B. Y. A numerical method for solving distributed order diffusion equations. Applied Mathematics Letters, 53, 92-99, (2016).

[17] Aminikhah, H., Refahi Sheikhani, A. ve Rezazadeh, H. Stability analysis of distributed order fractional Chen system. The Scientific World Journal, 1-13, (2013).

[18] Najafi, H. S., Sheikhani, A. R. ve Ansari, A. Stability analysis of distributed order fractional differential equations. In Abstract and Applied Analysis, Hindawi, (2011).

[19] Refahi, A., Ansari, A., Najafi, H. S. ve Merhdoust, F. Analytic study on linear systems of distributed order fractional differential equations. Le Matematiche, 67(2), 3-13, (2012).

[20] Dorciak L. Numerical models for simulation the fractional order control systems, UEF-04-94, The Academy of Sciences, Institute of Experimental Physic,Kosice, Slovak Republic, (1994).

[21] Meerschaert, M. M. ve Tadjeran, C. Finite difference approximations for fractional advection-dispersion flow equations. Journal of computational and applied mathematics, 172(1), 65-77, (2004).

[22] Mickens, R. E. Exact solutions to a finite-difference model of a nonlinear reactionadvection equation: Implications for numerical analysis. Numerical Methods for Partial Differential Equations, 5(4), 313-325, (1989).

[23] Mickens, R. E. Nonstandard finite difference models of differential equations. World scientific, (1994).

[24] Mickens, R. E. Applications of nonstandard finite difference schemes. World Scientific, (2000).

[25] Mickens, R. E. Nonstandard finite difference schemes for differential equations. Journal of Difference Equations and Applications, 8(9), 823-847, (2002).

[26] Oldham, K. ve Spanier, J. The fractional calculus theory and applications of differentiation and integration to arbitrary order. Elsevier, (1974).

[27] Podlubny, I. Fractional differential equations, vol. 198 of Mathematics in Science and Engineering, (1999).

[28] Mickens, R. E. Calculation of denominator functions for nonstandard finite difference schemes for differential equations satisfying a positivity condition. Numerical Methods for Partial Differential Equations: An International Journal, 23(3), 672-691, (2007).

[29] Ongun, M. Y. ve Turhan, I. A numerical comparison for a discrete HIV infection of CD4+ T-Cell model derived from nonstandard numerical scheme. Journal of Applied Mathematics, 2013, 1-9, (2013).

[30] Modanlı, M. Kesirli telegraf kısmi diferansiyel denklemlerin fark şeması metodu ile nümerik çözümü. Balıkesir Üniversitesi Fen Bilimleri Enstitüsü Dergisi, 20(1), 440-449, (2018).

[31] Ongun, M. Y., Arslan, D. ve Garrappa, R. Nonstandard finite difference schemes for a fractional order Brusselator system. Advances in Difference equations, 2013(1), 102, (2013).

[32] Hiçdurmaz, B. Numerical analysis for coupled systems of two-dimensional timespace fractional Schrödinger equations with trapping potentials. Balıkesir Üniversitesi Fen Bilimleri Enstitüsü Dergisi, 22(1), 1-12, (2020). 
[33] Dimitrov, D. T. ve Kojouharov, H. V. Nonstandard finite-difference methods for predator prey models with general functional response. Mathematics and Computers in Simulation, 78(1), 1-11, (2008).

[34] Elaydi S.N. An introduction to difference equations, Second edition, Undergraduate Texts in Mathematics, Springer-Verlag, New York, 2.3, 3, (1999).

[35] Dimitrov, D. T. ve Kojouharov, H. V. Nonstandard numerical methods for a class of predator-prey models with predator interference. Electronic Journal of Differential Equations (EJDE), 67-75, (2007).

[36] Ogata, K. Discrete time control systems. Englewood Cliffs, NJ: Prentice Hall, (1995).

[37] Ahmad, A., Javeed, N., Farman, M., Ahmad, M. O., Hafeez, A. ve Raza, A. Dynamical Behavior of Fractional Order SVIR Epidemic Model. International Journal of Analysis and Applications, 17(2), 260-274, (2019). 